\title{
A phase-field model for cohesive fracture
}

\author{
Clemens V. Verhoosel ${ }^{1, *}$ and René de Borst ${ }^{2}$ \\ ${ }^{1}$ Department of Mechanical Engineering, Eindhoven University of Technology, 5600 MB Eindhoven, Netherlands \\ ${ }^{2}$ School of Engineering, University of Glasgow, Glasgow G12 8LT, UK
}

\begin{abstract}
SUMMARY
In this paper a phase-field model for cohesive fracture is developed. After casting the cohesive zone approach in an energetic framework, which is suitable for incorporation in phase-field approaches, the phase-field approach to brittle fracture is recapitulated. The approximation to the Dirac function is discussed with particular emphasis on the Dirichlet boundary conditions that arise in the phase-field approximation. The accuracy of the discretisation of the phase field, including the sensitivity to the parameter that balances the field and the boundary contributions, is assessed at the hand of a simple example. The relation to gradientenhanced damage models is highlighted and some comments on the similarities and the differences between phase-field approaches to fracture and gradient-damage models are made. A phase-field representation for cohesive fracture is elaborated, starting from the above energetic framework. The strong as well as the weak formats are presented, the latter being the starting point for the ensuing finite element discretisation, which involves three fields: the displacement field, an auxiliary field which represents the jump in the displacement across the crack, and the phase field. Compared to phase-field approaches for brittle fracture, the modelling of the jump of the displacement across the crack is a complication, and the current work provides evidence that an additional constraint has to be provided in the sense that the auxiliary field must be constant in the direction orthogonal to the crack. The sensitivity of the results with respect to the numerical parameter needed to enforce this constraint is investigated, as well as how the results depend on the orders of the discretisation of the three fields. Finally, examples are given which demonstrate grid insensitivity for adhesive and for cohesive failure, the latter example being somewhat limited since only straight crack propagation is considered. Copyright (c) 2010 John Wiley \& Sons, Ltd.
\end{abstract}

Received . .

KEY WORDS: Cohesive Fracture, Phase-field models, Fracture, Cracking, Damage, Gradient models

\section{INTRODUCTION}

Two main approaches exist for the finite element modelling of fracture propagation in man-made and biological materials, namely the discrete crack models [1] and the smeared crack models [2]. In the discrete crack models fracture was originally assumed to occur when the nodal force that is normal to the element boundaries exceeds the maximum tensile force that can be sustained. New degrees-of-freedom at that node location are then created and a geometrical discontinuity is assumed to occur between the 'old' node and the newly created node. Two obvious drawbacks of the method are the continuous change of the topology of the discretisation and the restriction of the crack propagation to follow the mesh lines. Remeshing techniques [3,4] and advanced discretisation techniques such as meshless methods [5] or partition-of-unity based finite element methods $[6,7,8,9,10]$ can overcome these limitations. Yet, although sophisticated crack-front

*Correspondence to: Clemens V. Verhoosel, Department of Mechanical Engineering, Eindhoven University of Technology, P.O. Box 513, 5600 MB Eindhoven, Netherlands,

E-mail: c.v.verhoosel@tue.nl 
tracking methods have been developed, for instance based on level sets [11, 12], the robust and efficient analysis of three-dimensional problems can still pose problems.

The counterpart of the discrete crack concept is the smeared crack concept, in which a cracked solid is imagined to be a continuum where the notions of stress and strain apply. Upon cracking, the isotropic stress-strain relation is replaced by an orthotropic stress-strain relation which incorporates the effect of local material degradation. However, the topology of the original finite element mesh is preserved. This is computationally efficient and it is for this reason that the method has come into widespread use and has replaced discrete crack models in most large-scale computations.

Smeared-crack models can be cast within the framework of (anisotropic) continuum damage mechanics [13, 14], and thus share the disadvantages of damage models. A particular concern regarding damage evolution in standard continuum models is the inherent mesh sensitivity that occurs after reaching a certain damage level. This mesh sensitivity goes beyond the standard discretisation sensitivity of numerical approximation methods for partial differential equations and is not related to deficiencies in the discretisation methods. Instead, the underlying reason for this mesh sensitivity is a local change in character of the governing partial differential equations. This change of character leads to a loss of well-posedness of the initial/boundary value problem and results in an infinite number of possible solutions. After discretisation, a finite number of solutions results. For a finer discretisation, the number of solutions increases, which explains the observed spurious mesh sensitivity. Well-posedness can be (partially) restored in a variety of ways, including non-local averaging schemes [15], and implicit gradient damage models [16].

Not unrelated to implicit gradient damage approaches are the phase-field models for fracture. Pioneering work has been done by Francfort, Bourdin and Marigo [17, 18, 19], who proposed a phase-field approximation of the variational formulation for brittle fracture based on the MumfortShah potential [20]. More recently, Miehe and co-workers [21, 22, 23] have derived a phase-field formulation for brittle fracture based on thermodynamical considerations. This formulation closely resembles that in [18]. Both models have been applied to dynamic fracture [23, 24, 25]. An extension of the variational formulation for brittle fracture to cohesive fracture has been considered in [19], but a phase-field approximation is not yet available. It is the purpose of this paper to develop such an approximation.

The development of a phase-field model for cohesive fracture is a non-trivial extension of the model available for brittle fracture. In fact, considering well-established numerical methods for discrete models of brittle and cohesive fracture, it is observed that they rely on very different concepts. In this contribution we will explore the fundamental properties of cohesive zone formulations in the context of phase-field formulations. For achieving this, we will focus on adhesive interfaces, i.e. interfaces that do not propagate. Our developments work towards a model for propagating cohesive cracks of arbitrary topological complexity, but such a complete model is not fully covered in this contribution. Yet, we will present first results for a simple test case with a propagating interface in the final part of the paper.

The paper is structured as follows. In Section 2 the cohesive phase-field model will be derived, and we will first consider cohesive fracture in its original, discrete form. Next, we introduce the phase field approach and apply it to obtain a phase-field model for cohesive fracture. In Section 3 we consider the finite element discretization of the model. A series of one and two-dimensional test cases is considered in Section 4, while concluding remarks are made in Section 5.

\section{COHESIVE FRACTURE AND PHASE FIELDS}

\subsection{Cohesive fracture}

We consider a volume $\Omega$ with an internal discontinuity boundary $\Gamma$ as shown in Figure 1a. The position of a material point is determined by the coordinate $\boldsymbol{x}$ in a Cartesian reference frame. Displacement and traction components are prescribed along disjoint parts of the external boundary of the domain, $\partial \Omega_{g_{i}}$ and $\partial \Omega_{h_{i}}$, respectively, with $\boldsymbol{g}$ and $\boldsymbol{h}$ the Dirichlet and Neumann data. As the starting point for the derivation of the phase-field approximation to cohesive fracture models, we 


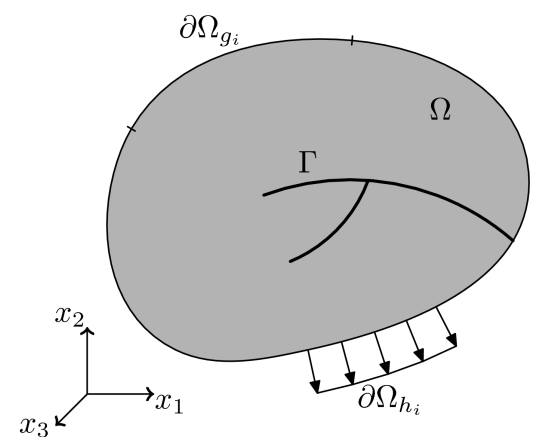

(a) Discrete interface

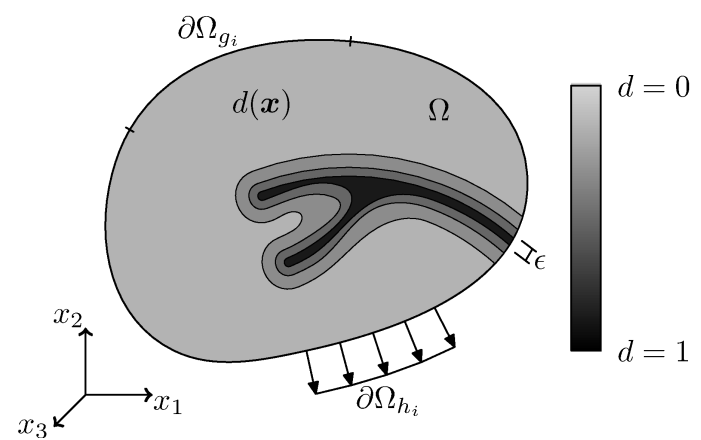

(b) Smeared interface

Figure 1. Schematic representation of a solid domain with (a) a discrete internal discontinuity interface and (b) a smeared interface

first consider the potential energy for the case of a discrete description of brittle fracture (e.g. [17]):

$$
\Psi_{\text {pot }}=\int_{\Omega} \psi^{e}(\varepsilon) \mathrm{d} V+\int_{\Gamma} \mathcal{G}_{c} \mathrm{~d} A
$$

In this expression, the elastic energy density $\psi^{e}$ is a function of the infinitesimal strain tensor $\varepsilon$, with components

$$
\varepsilon_{i j}=u_{(i, j)}=\frac{1}{2}\left(\frac{\partial u_{i}}{\partial x_{j}}+\frac{\partial u_{j}}{\partial x_{i}}\right)
$$

where $\boldsymbol{u}$ is the displacement field. Under the assumption of small displacement gradients, this infinitesimal strain tensor is an appropriate measure of the deformation of the body. The elastic energy density is expressed by Hooke's law for an isotropic linear elastic material as

$$
\psi^{e}(\varepsilon)=\frac{1}{2} \lambda \varepsilon_{i i} \varepsilon_{j j}+\mu \varepsilon_{i j} \varepsilon_{i j},
$$

with $\lambda$ and $\mu$ the Lamé constants. In Equation (1) the fracture energy, i.e. the amount of energy dissipated upon the creation of a unit of fracture surface) is denoted by $\mathcal{G}_{c}$. The potential energy $\Psi_{\text {pot }}$ governs the balance between elastic energy in the bulk material and the fracture energy. These two energy terms are evidently different in nature as the internal energy is recoverable, while the fracture energy is dissipative. Variational formulations for brittle fracture incorporate this fundamental difference by means of an irreversibility condition, which enforces that cracks can only nucleate and propagate, and not heal (e.g. [19]).

The essential difference between brittle and cohesive fracture models is the dependence of the fracture energy on the crack opening for the latter type of models. While in the former class of models the fracture energy $\mathcal{G}_{c}$ is instantly dissipated upon the creation of a unit fracture surface, in the cohesive case the energy is released gradually, and the energy dissipation is governed by a fracture energy function:

$$
\mathcal{G}=\mathcal{G}(\llbracket \boldsymbol{u} \rrbracket, \kappa)
$$

which depends on the jump of the displacement field $\llbracket \boldsymbol{u} \rrbracket$ across the discontinuity $\Gamma$ and on a history parameter $\kappa$ (or, more generally, a set of history parameters). The history parameter obeys the KuhnTucker conditions to distinguish between loading and unloading. The cohesive tractions are obtained through differentiation of the fracture energy with respect to the crack opening:

$$
\boldsymbol{t}=\frac{\partial \mathcal{G}}{\partial \llbracket \boldsymbol{u} \rrbracket} \quad \rightarrow \quad t=t(\llbracket \boldsymbol{u} \rrbracket, \kappa)
$$

Both adhesive (initially elastic) and cohesive (initially rigid) traction-opening relations can be constructed by means of the fracture energy function (4). Examples of both cases are shown in 

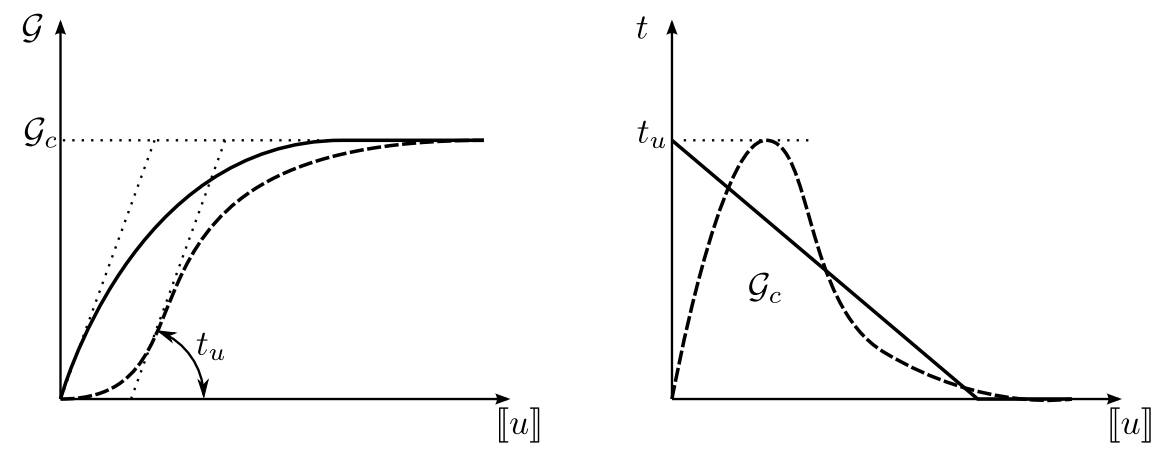

Figure 2. Initially rigid (solid line) and initially elastic (dashed line) crack-opening relations

Figure 2. The most important parameters of the crack-opening relations are the fracture energy, $\mathcal{G}_{c}$, which corresponds to the value of the fracture energy function $\mathcal{G}$ at full crack opening (or, equivalently, to the total area under the traction-opening curve), and the fracture strength $t_{u}$. The latter parameter sets the maximum value of the traction-opening law or, equivalently, the maximum slope in the fracture energy function. From the fracture energy function for adhesive interfaces, the dashed line in Figure 2, it is observed that at the fracture strength the fracture energy function changes from convex to concave, which reflects the change in the character of the underlying set of differential equations.

Similar to brittle fracture, but now using the fracture energy function (4), the potential energy for the description of discrete cohesive fracture is written as:

$$
\Psi_{\text {pot }}=\int_{\Omega} \psi^{e}(\varepsilon) \mathrm{d} V+\int_{\Gamma} \mathcal{G}(\llbracket \boldsymbol{u} \rrbracket, \kappa) \mathrm{d} A
$$

A classical result is recovered when the variation of the potential energy is considered:

$$
\delta \Psi_{\text {pot }}=\int_{\Omega} \sigma_{i j}(\varepsilon) \delta \varepsilon_{i j} \mathrm{~d} V+\int_{\Gamma} t_{i}(\llbracket \boldsymbol{u} \rrbracket, \kappa) \delta \llbracket u_{i} \rrbracket \mathrm{d} A
$$

From this expression the Cauchy stress is defined as

$$
\boldsymbol{\sigma}=\frac{\partial \psi^{e}}{\partial \varepsilon}
$$

which, for isotropic linear elasticity, specializes to:

$$
\sigma_{i j}=\lambda \varepsilon_{k k} \delta_{i j}+2 \mu \varepsilon_{i j}
$$

Equation (7) is the familiar decomposition of the variation of internal energy into a part related to the continuum and a part associated with the cohesive interfaces.

Before we proceed with the derivation of the phase-field model for cohesive fracture we stipulate the fundamentally different dissipation mechanisms for brittle and for cohesive fracture. Whereas energy dissipation in the brittle case is directly related to the creation of an internal discontinuity surface, in the cohesive case there is the requirement that a crack surface further opens. This distinction is very clear for adhesive interfaces. For such interfaces a geometric discontinuity is present from the onset, but energy is only dissipated upon opening of the interface. In order to develop a phase-field model for cohesive fracture it is crucial to properly include the dissipative mechanism in the energy balance equations. Compared to the models for brittle fracture it is required to model crack opening, since it is the kinematic quantity that drives energy dissipation.

\subsection{Phase field formulation}

The idea of phase-field models is to represent a discrete interface by means of a continuous, or smeared, field. In the case of the cohesive zone formulation discussed in the previous section, a 


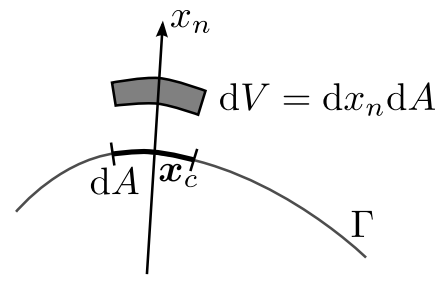

Figure 3. Zoom of the discontinuity surface $\Gamma$ with definitions of the infinitesimal area $\mathrm{d} A$ and infinitesimal volume $\mathrm{d} V$

phase field can be used to create a smeared approximation, $\Gamma_{\epsilon}$, of the discrete interface $\Gamma$. It is noted that the subscript $\epsilon$ is used to refer to a smeared approximation. Considering the potential energy functional, equation (6), the first step towards a phase-field description is the representation of the infinitesimal surface area $\mathrm{d} A$ by a smeared approximation $\mathrm{d} A_{\epsilon}$. Since this approximation is smeared out over the volume the surface area must be reformulated as a volume integral. Using a Dirac function, the infinitesimal surface area $\mathrm{d} A$ at every point $\boldsymbol{x}_{c}$ of the fracture surface, can be written as:

$$
\mathrm{d} A\left(\boldsymbol{x}_{c}\right)=\underbrace{\int_{x_{n}=-\infty}^{\infty} \delta\left(x_{n}\right) \mathrm{d} x_{n}}_{=1} \mathrm{~d} A=\int_{x_{n}=-\infty}^{\infty} \delta\left(x_{n}\right) \mathrm{d} V,
$$

with $x_{n}=\left(\boldsymbol{x}-\boldsymbol{x}_{c}\right) \cdot \boldsymbol{n}\left(\boldsymbol{x}_{c}\right)$ and $\boldsymbol{n}(\boldsymbol{x})$ the unit vector normal to the fracture surface (see Figure 3). Equation (10) directly allows for smeared descriptions of the fracture surface by employing a smeared approximation of the Dirac function. As in [21, 22] we consider the approximated Dirac function

$$
\delta_{\epsilon}\left(x_{n}\right)=\frac{1}{2 \epsilon} \exp \left(-\frac{\left|x_{n}\right|}{\epsilon}\right)
$$

with $\epsilon>0$ a length scale parameter. Evidently, for arbitrary $\epsilon$, it holds that:

$$
\int_{-\infty}^{\infty} \delta_{\epsilon}\left(x_{n}\right) \mathrm{d} x_{n}=1
$$

From equation (11) it is observed that for higher values of $\epsilon$ the fracture surface is distributed over a larger volume. Indeed, $\epsilon$ is directly related to the 'width' of the smeared interface. Upon substitution of equation (11) into equation (10) the smeared infinitesimal fracture surface area follows as:

$$
\mathrm{d} A_{\epsilon}\left(\boldsymbol{x}_{c}\right)=\int_{x_{n}=-\infty}^{\infty} \delta_{\epsilon}\left(x_{n}\right) \mathrm{d} V
$$

A fundamental problem with the smeared Dirac function approximation, equation (11), is that it is not straightforward to generalize it to more dimensions. Therefore, rather than using this approximate function directly, it is obtained implicitly through the solution of the boundary value problem

$$
\begin{cases}d-4 \epsilon^{2} \frac{\mathrm{d}^{2} d}{\mathrm{~d} x_{n}^{2}}=0 & x_{n} \in \mathbb{R} \backslash 0 \\ d=1 & x_{n}=0 \\ d=0 & x_{n}= \pm \infty\end{cases}
$$

with $d\left(x_{n}\right) \in[0,1]$ a scalar field, which equals 1 at the centre of the discontinuity, i.e. for $x_{n}=0$, and vanishes for $x_{n}= \pm \infty$. The approximate Dirac function, equation (11), then follows as

$$
\delta_{\epsilon}=\frac{d^{2}}{4 \epsilon}+\epsilon\left(\frac{\mathrm{d} d}{\mathrm{~d} x_{n}}\right)^{2}
$$

It is noted that the approximate Dirac function would also follow from the simpler expression:

$$
\delta_{\epsilon}=\frac{d^{2}}{2 \epsilon}
$$


Using the expression of equation (15), however, has the advantage that upon substitution into equation (12) and multiplication by a fracture energy we obtain an energy functional, and minimisation of this energy functional then yields the differential equation of equation (14).

In [21] it has been demonstrated how equation (14) allows for the description of fracture surfaces in a two-dimensional setting. In the brittle fracture description, however, the Dirichlet constraints are rendered unnecessary due to the appearance of thermodynamic driving force terms. A similar concept has been used in [25] for the representation of fracture surfaces that are a priori present in the mesh, thereby avoiding the necessity of introducing (mesh-dependent) Dirichlet constraints. Here, we follow this line and weakly impose the Dirichlet constraints by adding the constraint term

$$
d(0)=1
$$

in a weighted sense to the weak form of the boundary value problem (14). Using the test function $v$ the weak form is obtained as

$$
\int_{-\infty}^{\infty} v\left(d-4 \epsilon^{2} \frac{\mathrm{d}^{2} d}{\mathrm{~d} x_{n}^{2}}\right) \mathrm{d} x_{n}+C \frac{4 \epsilon^{2}}{h} \int_{-\infty}^{\infty} v(d-1) \delta\left(x_{n}\right) \mathrm{d} x_{n}=0,
$$

with $C$ a positive constant that balances the weight of the differential equation $v s$ that of the boundary condition, and $h$ a parameter proportional to the mesh size. Note that the multiplication of the second integral by a factor 4 is arbitrary, and influences the choice of the constant $C$. We rewrite equation (17) as

$$
\int_{-\infty}^{\infty} v\left(d-4 \epsilon^{2} \frac{\mathrm{d}^{2} d}{\mathrm{~d} x_{n}^{2}}+C \frac{4 \epsilon^{2}}{h}(d-1) \delta\left(x_{n}\right)\right) \mathrm{d} x_{n}=0,
$$

and obtain after a slight rewriting:

$$
(1+\mathcal{F}) d-4 \epsilon^{2} \frac{\mathrm{d}^{2} d}{\mathrm{~d} x_{n}^{2}}=\mathcal{F}
$$

with the "driving force" term given by

$$
\mathcal{F}=C \frac{4 \epsilon^{2}}{h} \delta\left(x_{n}\right)
$$

From equation (19) we directly observe the dependence of the phase-field on the driving force $\mathcal{F}$. If $\mathcal{F} \ll 1$, the phase-field parameter $d$ approaches zero, whereas if $\mathcal{F} \gg 1, d$ goes to one. In order to allow for numerical integration of the constraint term, we approximate the Dirac function as:

$$
\delta\left(x_{n}\right) \approx \begin{cases}\frac{1}{h}\left(1-\frac{\left|x_{n}\right|}{h}\right) & -h \leq x_{n} \leq h \\ 0 & \text { otherwise }\end{cases}
$$

This Dirac approximation is not directly related to the earlier approximation for the smeared interface, $\delta_{\epsilon}$, equation (11).

In Figure 4 we show the convergence of the linear finite element approximation of the differential equation (19) on a domain $[-L / 2, L / 2] \subset \mathbb{R}$ with $L=1$ and with $\epsilon=\frac{L}{20}$. The approximate solution is obtained using the weak form of equation (19)

$$
\int_{-L}^{L}\left[v(1+\mathcal{F}) d+4 \epsilon^{2} \frac{\mathrm{d} v}{\mathrm{~d} x_{n}} \frac{\mathrm{d} d}{\mathrm{~d} x_{n}}\right] \mathrm{d} x_{n}=\int_{-L}^{L} v \mathcal{F} \mathrm{d} x_{n},
$$

where use has been made of integration by parts. Note that, formally, an additional term should be present that accommodates the non-smooth parameters in the differential equation at $x_{n}=0$. However, removal of this term results in a more smooth solution at the centre of the crack, and in practice, this term appears to be of minor influence.

In Figure 4 (left) the discretisation accuracy of the phase field is measured in the $L^{2}$-error with respect to the exact solution of the set of equations (14). As a consequence of the discrete domain 

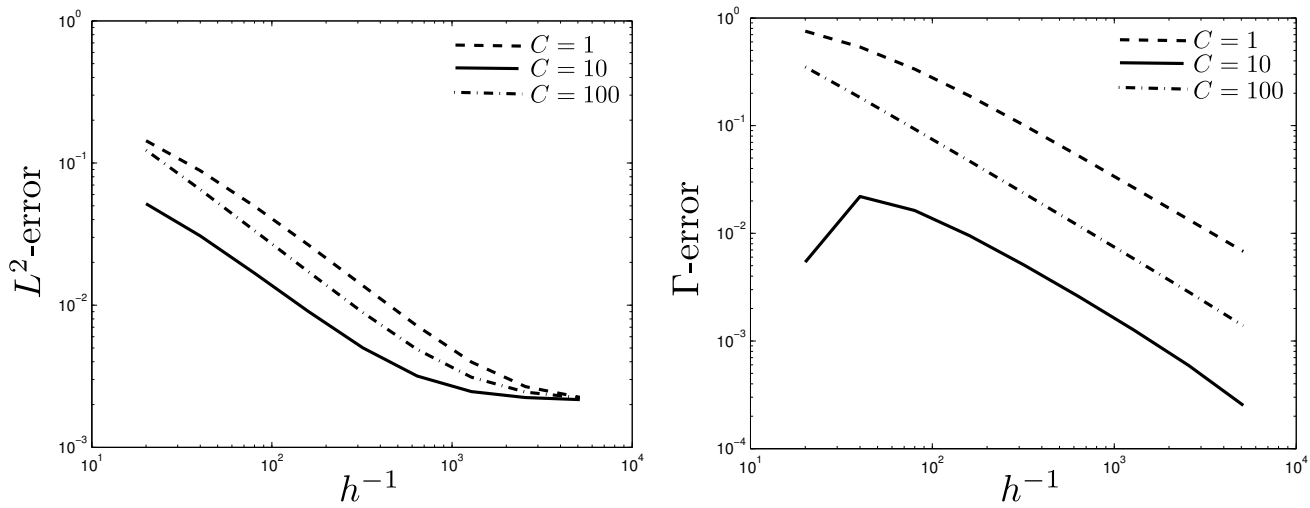

Figure 4. Mesh convergence of the field $d$ in the $L^{2}$-error (left) and the $\Gamma$-error (right) for various intensities of the forcing term $C$
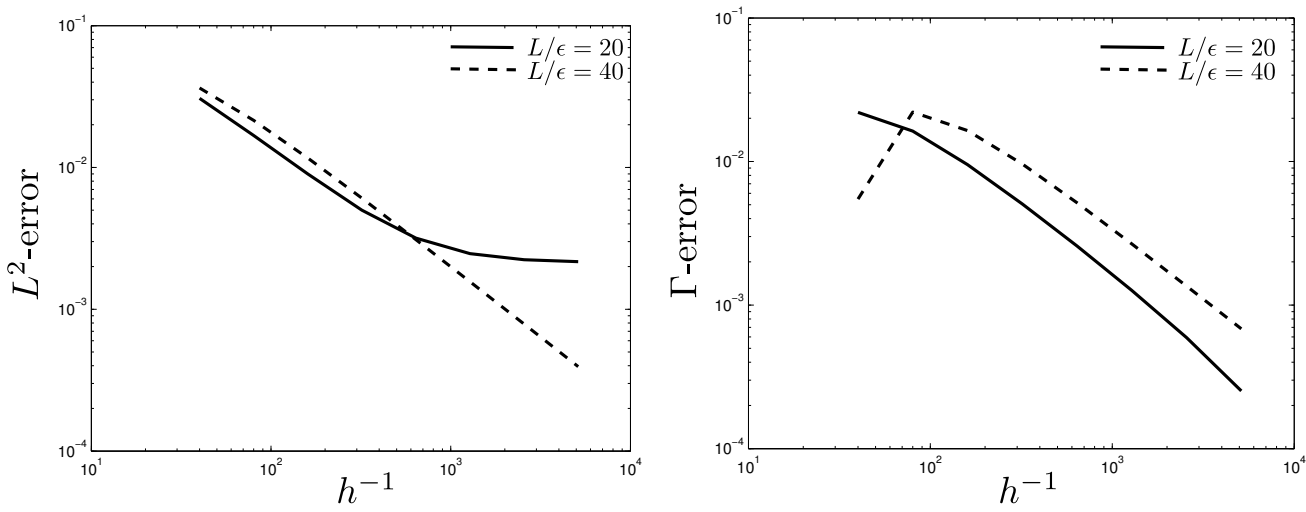

Figure 5. Mesh convergence of the field $d$ in the $L^{2}$-error (left) and the $\Gamma$-error (right) for various settings of the length scale parameter $\epsilon$ with $C=10$

being finite, the phase-field solution $d(x)$ does not converge to the exact solution of the problem, equation (14). This is confirmed by the convergence behaviour of the same problem, but with half the value of the length scale $\epsilon$, which is given in Figure 5. From Figure 4 it is observed that the dimensionless coefficient $C$ is of minor importance for the rate of convergence of the interface error. However, the magnitude of the error for a fixed mesh size is dependent on this coefficient. In the remainder we will employ $C=10$. In Figure 4 and in Figure 5 we also give the convergence behaviour measured in the $\Gamma$-error, defined as:

$$
\mathcal{E}_{\Gamma}=\left|\int_{-L / 2}^{L / 2} \delta_{\epsilon} \mathrm{d} x-1\right|
$$

Asymptotic convergence of this measure for the error is observed for both $\epsilon=L / 20$ and $\epsilon=L / 40$.

The implicit definition of the smeared interface $\Gamma_{\epsilon}$ as derived for a one-dimensional setting can be extended straightforwardly to more dimensions. The phase-field parameter $d$ then follows from the solution of the weak form problem:

$$
\int_{\Omega}\left[v(1+\mathcal{F}) d+4 \epsilon^{2} \frac{\partial v}{\partial x_{i}} \frac{\partial d}{\partial x_{i}}\right] \mathrm{d} V=\int_{\Omega} v \mathcal{F} \mathrm{d} V \quad \forall v
$$


In this expression, the thermodynamic driving force that sets the crack is still given by (20), with $x_{n}=\left(\boldsymbol{x}-\boldsymbol{x}_{c}\right) \cdot \boldsymbol{n}\left(\boldsymbol{x}_{c}\right)$ and $\boldsymbol{x}_{c}(\boldsymbol{x})=\underset{\boldsymbol{y} \in \Gamma}{\operatorname{argmin}}(\|\boldsymbol{y}-\boldsymbol{x}\|)$. Once the phase-field over a multidimensional domain is determined, the approximate Dirac function (equation (15) in the onedimensional setting), is found as

$$
\delta_{\epsilon}=\frac{d^{2}}{4 \epsilon}+\epsilon \frac{\partial d}{\partial x_{i}} \frac{\partial d}{\partial x_{i}},
$$

which, in conjunction with equation (13) yields an approximation of an infinitesimal surface area over the crack surface.

\section{Remark}

- Relation to the implicit gradient damage formulation Equation (24), rewritten in its strong form reads:

$$
(1+\mathcal{F}) d-4 \epsilon^{2} \frac{\partial^{2} d}{\partial x_{i}^{2}}=\mathcal{F} .
$$

This equation serves the same purpose as the differential equation for the equivalent strain in implicit gradient damage models [16], which also smoothens the solution. The relation to gradient damage formulations in which the local damage variable $\tilde{d}$ is smoothened through

$$
d-4 \epsilon^{2} \frac{\partial^{2} d}{\partial x_{i}^{2}}=\tilde{d}
$$

to give a non-local, or averaged damage value $d$, is even more clear [26, 27]. Comparing equation (27) with equation (26) reveals a crucial difference. When $d \rightarrow 1, \mathcal{F}$ becomes much larger than 1. As a consequence, $(1+\mathcal{F}) d$, the first term in equation (26), becomes dominant, and the smearing, or distributing term gradually vanishes. A similar effect can be achieved in implicit gradient damage models by letting the internal length scale parameter go to zero upon the accumulation of damage [28].

\subsection{Phase-field representation for cohesive fracture}

With the cohesive zone formulation introduced in Section 2.1 and the phase-field approximation of the interface discussed in Section 2.2, the main ingredients for the formulation of the phase-field cohesive zone formulation are now present. The main result from the previous section is that the infinitesimal unit surface area, $\mathrm{d} A$, can be approximated by the smeared measure $\mathrm{d} A_{\epsilon}$, such that an integral of some function $g(\boldsymbol{x})$ over the surface $\Gamma$ can be expressed as

$$
\int_{\Gamma} g(\boldsymbol{x}) \mathrm{d} A \approx \int_{\Omega} g(\boldsymbol{x}) \delta_{\epsilon} \mathrm{d} V=\int_{\Omega} g\left(\frac{d^{2}}{4 \epsilon}+\epsilon \frac{\partial d}{\partial x_{i}} \frac{\partial d}{\partial x_{i}}\right) \mathrm{d} V,
$$

where it is noted that the unit of $\delta_{\epsilon}$ is $[1 / \mathrm{m}]$. We use this approximation to rewrite the surface integral in equation (6) to obtain an expression for the potential energy with the contribution of the fracture interface smeared over the domain by the phase field:

$$
\Psi_{\text {pot }, \epsilon}=\int_{\Omega}\left(\psi^{e}\left(\varepsilon^{e}\right)+\mathcal{G}(\boldsymbol{v}, \kappa) \delta_{\epsilon}\right) \mathrm{d} V
$$

Compared to equation (6), we have replaced the infinitesimal strain tensor $\varepsilon$ by the 'elastic' infinitesimal strain tensor $\boldsymbol{\varepsilon}^{e}$, and the jump in the displacement field $\llbracket \boldsymbol{u} \rrbracket\left(\boldsymbol{x}_{c}\right)$ with an auxiliary field $\boldsymbol{v}(\boldsymbol{x})$. These adjustments are necessary since in the phase-field formulation the crack only exists in a smeared sense. Consequently, the clear distinction between the bulk and interface kinematics, i.e. between the infinitesimal strain tensor, equation (2), and the crack opening $\llbracket \boldsymbol{u} \rrbracket$ is lost in the vicinity of the crack. The remainder of this section will focus on the derivation of proper expressions for these kinematic quantities.

In the phase-field formulation for cohesive fracture it is crucial to derive kinematic relations that are consistent with the discrete problem, in the sense that as the length scale parameter $\epsilon$ approaches 
zero, the kinematics of the discrete problem are recovered. In order to arrive at such relations, we first introduce the distributed internal discontinuity

$$
\Gamma_{\epsilon}=\{\boldsymbol{x} \in \Omega \mid d(\boldsymbol{x})>\text { tol }\}
$$

with tol $\ll 1$ a small tolerance that truncates the support of the smeared crack. The smeared internal discontinuity $\Gamma_{\epsilon}$ provides the support for the auxiliary field $\boldsymbol{v}(\boldsymbol{x})$. Hence, the auxiliary field is only present at the smeared crack, and the kinematics away from the smeared crack are fully governed by the displacement field $\boldsymbol{u}$. Next, we define the normal to the smeared crack based on the point closest to the discrete boundary $\Gamma$, denoted by $\boldsymbol{x}_{c}$, as

$$
\boldsymbol{n}(\boldsymbol{x})=\boldsymbol{n}\left(\boldsymbol{x}_{c}\right) \quad \text { with } \quad \boldsymbol{x}_{c}(\boldsymbol{x})=\underset{\boldsymbol{y} \in \Gamma}{\operatorname{argmin}}(\|\boldsymbol{y}-\boldsymbol{x}\|)
$$

In the discrete formulation, the displacement jump is strictly defined at the internal discontinuity $\Gamma$. In the phase-field approach the crack exists in smeared sense, and so does the crack opening. Therefore, we approximate the discrete displacement jump at $\boldsymbol{x}_{c}$ in terms of the auxiliary jump field $v$ as

$$
\llbracket \boldsymbol{u} \rrbracket\left(\boldsymbol{x}_{c}\right) \approx \int_{-\infty}^{\infty} \boldsymbol{v}(\boldsymbol{x}) \delta_{\epsilon} \mathrm{d} x_{n}
$$

Under the condition that the auxiliary field is constant in the direction normal to the fracture, i.e.

$$
\frac{\partial \boldsymbol{v}}{\partial x_{n}}=\mathbf{0}
$$

we have

$$
\boldsymbol{v}(\boldsymbol{x})=\boldsymbol{v}\left(\boldsymbol{x}_{c}+x_{n} \boldsymbol{n}\right)=\boldsymbol{v}\left(\boldsymbol{x}_{c}\right)
$$

with $x_{n}$ the coordinate along $\boldsymbol{n}$. Back-substitution into equation (32) yields

$$
\llbracket \boldsymbol{u} \rrbracket\left(\boldsymbol{x}_{c}\right) \approx \int_{-\infty}^{\infty} \boldsymbol{v}\left(\boldsymbol{x}_{c}\right) \delta_{\epsilon} \mathrm{d} x_{n}=\boldsymbol{v}\left(\boldsymbol{x}_{c}\right) \int_{-\infty}^{\infty} \delta_{\epsilon} \mathrm{d} x_{n}=\boldsymbol{v}\left(\boldsymbol{x}_{c}\right),
$$

which shows that $\boldsymbol{v}(\boldsymbol{x})$ reflects the crack opening at the closest point $\boldsymbol{x}_{c}$ on the discrete internal boundary. Consequently, in equation (29) the crack opening $\llbracket \boldsymbol{u} \rrbracket$ that appears as an argument of the fracture energy can be directly replaced by the auxiliary field $\boldsymbol{v}$. In the limiting case that the length scale parameter $\epsilon$ goes to zero, the smeared crack $\Gamma_{\epsilon}$ coincides with the discrete crack $\Gamma$ and the auxiliary displacement field coincides with the discrete displacement jump. The requirement that the auxiliary jump field is constant in the direction normal to the crack is enforced weakly through the addition of a penalty term in the internal energy functional:

$$
\Psi_{\text {pot }, \epsilon}=\int_{\Omega}\left(\psi^{e}\left(\varepsilon^{e}\right)+\mathcal{G}(\boldsymbol{v}, \kappa) \delta_{\epsilon}+\frac{1}{2} \alpha\left|\frac{\partial \boldsymbol{v}}{\partial x_{n}}\right|^{2}\right) \mathrm{d} V
$$

with $\alpha$ a positive constant. In Section 2.4 we will further comment on the influence of this parameter when considering the strong form problem.

With the discontinuity kinematics determined through the auxiliary field $\boldsymbol{v}$, the elastic strain tensor $\varepsilon^{e}$ can be derived by requiring the auxiliary field not to exert external power on the problem, such that the balance of power is given by:

$$
\int_{\Omega}\left(\sigma_{i j} \dot{\varepsilon}_{i j}^{e}+t_{i}(\boldsymbol{v}, \kappa) \delta_{\epsilon} \dot{\theta}_{i}\right) \mathrm{d} V=\int_{\partial \Omega} h_{i} \dot{u}_{i} \mathrm{~d} A
$$

with $\boldsymbol{h}$ representing the traction along the Neumann boundary $\partial \Omega_{h_{i}}$. Applying Gauss' theorem to the right-hand side of this equation, and requiring the traction to be in equilibrium with the Cauchy stress, $\sigma_{i j} n_{j}=h_{i}$, yields

$$
\int_{\Omega} \sigma_{i j}\left(\dot{\varepsilon}_{i j}^{e}+\delta_{\epsilon} \operatorname{sym}\left(\dot{v}_{i} n_{j}\right)\right) \mathrm{d} V=\int_{\Omega} \sigma_{i j} \dot{u}_{(i, j)} \mathrm{d} V
$$


From this power balance it is directly observed that the elastic infinitesimal strain tensor, $\varepsilon^{e}$, is related to the displacement field $\boldsymbol{u}$ and auxiliary field $\boldsymbol{v}$ as

$$
\varepsilon_{i j}^{e}=u_{(i, j)}-\operatorname{sym}\left(v_{i} n_{j}\right) \delta_{\epsilon} .
$$

In this expression the first part is the symmetric part of the gradient of the displacement field. The second part can be interpreted as the strain caused by the displacement jump. Hence, the elastic strain is defined as the symmetric gradient of the displacement field, minus the strain caused by the crack opening. We immediately note from equation (39) that away from the crack, i.e. for $\boldsymbol{x} \notin \Gamma_{\epsilon}$, the continuum expression of equation (2) is recovered. In the limiting case that $\epsilon$ goes to zero, the elastic equivalent strain is equal to the symmetric gradient of the displacement field at every point in the interior of the domain. The unbounded strain term at the discontinuity is caused by the jump of the displacement field over this internal boundary.

With the kinematics for the displacement jump and elastic strain defined, the weak form of the cohesive phase-field problem is obtained by taking an arbitrary variation in the displacement field $\boldsymbol{u}$ and displacement jump field $\boldsymbol{v}$, while keeping the phase-field constant

$$
\delta \Psi_{\text {pot }, \epsilon}=\int_{\Omega}\left(\sigma_{i j} \delta \varepsilon_{i j}^{e}+\delta_{\epsilon} t_{i}(\boldsymbol{v}, \kappa) \delta v_{i}+\alpha \frac{\partial v_{i}}{\partial x_{n}} \frac{\partial \delta v_{i}}{\partial x_{n}}\right) \mathrm{d} V=\delta \Psi_{\text {ext }}
$$

where $\delta \Psi_{\text {ext }}$ is the variation in external work, which, in the absence of body forces can be written as

$$
\delta \Psi_{\text {ext }}=\int_{\partial \Omega} h_{i} \delta u_{i} \mathrm{~d} S
$$

The variation in the elastic strain is derived from equation (39) as

$$
\delta \varepsilon_{i j}^{e}=\delta u_{(i, j)}-\operatorname{sym}\left(\delta v_{i} n_{j}\right) \delta_{\epsilon} .
$$

\subsection{Strong form of the boundary value problem}

In order to gain more insight into the phase-field formulation for cohesive fracture, we rewrite the weak formulation of equation (40) into a strong format. In order to derive the strong form we substitute equation (42) into equation (40) to obtain:

$$
\int_{\Omega}\left(\sigma_{i j} \delta u_{(i, j)}-\delta_{\epsilon} \sigma_{i j} \operatorname{sym}\left(\delta v_{i} n_{j}\right)+\delta_{\epsilon} t_{i}(\boldsymbol{v}, \kappa) \delta v_{i}+\alpha \frac{\partial v_{i}}{\partial x_{n}} \frac{\partial \delta v_{i}}{\partial x_{n}}\right) \mathrm{d} V=\int_{\partial \Omega} h_{i} \delta u_{i} \mathrm{~d} S
$$

After some manipulations this equation can be rewritten as

$$
\begin{array}{r}
\int_{\Omega}\left(-\frac{\partial \sigma_{i j}}{\partial x_{j}} \delta u_{i}-\left[\delta_{\epsilon}\left(\sigma_{i j} n_{j}-t_{i}(\boldsymbol{v}, \kappa)\right)+\alpha \frac{\partial^{2} v_{i}}{\partial x_{n}^{2}}\right] \delta v_{i}\right) \mathrm{d} V \\
=\int_{\partial \Omega}\left(h_{i}-\sigma_{i j} n_{j}\right) \delta u_{i} \mathrm{~d} S-\int_{\partial \Gamma_{\epsilon}} \alpha \frac{\partial v_{i}}{\partial x_{n}} \delta v_{i} \mathrm{~d} S,
\end{array}
$$

where it is assumed that the curvature of the crack is small. Considering a variation in the displacement field then yields the strong form boundary value problem:

$$
\begin{cases}\frac{\partial \sigma_{i j}}{\partial x_{j}}=0 & \boldsymbol{x} \in \Omega \\ \sigma_{i j} n_{j}=h_{i} & \boldsymbol{x} \in \partial \Omega\end{cases}
$$

As can be seen, from the perspective of the displacement field, the phase-field cohesive zone formulation is a regular continuum mechanics problem, supplemented with the standard constitutive relation, equation (9), and the phase-field dependent strain measure $\varepsilon^{e}$, equation (39).

Considering a variation in the displacement jump field in equation (44) yields the additional boundary value problem:

$$
\begin{cases}\delta_{\epsilon}\left[t_{i}(\boldsymbol{v}, \kappa)-\sigma_{i j} n_{j}\right]=\alpha \frac{\partial^{2} v_{i}}{\partial x_{n}^{2}} & \boldsymbol{x} \in \Gamma_{\epsilon} \\ \frac{\partial v_{i}}{\partial x_{n}}=0 & \boldsymbol{x} \in \partial \Gamma_{\epsilon}\end{cases}
$$


The interpretation of this differential equation is that it enforces the equilibrium of forces over the smeared interface. In fact, in the limiting case of $\epsilon$ going to zero, the well known result $t_{i}(\llbracket \boldsymbol{u} \rrbracket, \kappa)=\sigma_{i j} n_{j}$ is recovered. The penalty term which enforces a constant crack opening field in the direction perpendicular to the crack can perturb this equilibrium. The magnitude of this perturbation depends on the parameter $\alpha$. The influence of this parameter on the results, will be addressed in Section 4.

\section{DISCRETISATION}

Since in this contribution we focus on the simulation of pre-defined interfaces, we employ a two stage solution algorithm. In the first step the phase field corresponding to the pre-defined crack pattern is computed. In the second step, the fracture behaviour of these interfaces upon mechanical loading of the specimen is solved using a standard incremental iterative solution procedure. In Section 4.4 we present a first numerical result that extends this two stage procedure for pre-defined cracks to a staggered scheme suitable for the simulation of propagating interfaces.

\subsection{Phase field discretisation}

The cohesive phase-field formulation is discretised using finite elements. For the discretisation of the weak form of equation (22) we consider the interpolation of the phase field as

$$
d(\boldsymbol{x})=\boldsymbol{N}_{d}(\boldsymbol{x}) \boldsymbol{a}_{d},
$$

where $\boldsymbol{a}_{d}$ is an array containing the nodal coefficients of the phase field and $\boldsymbol{N}_{d}$ contains the finite element basis functions. By differentiation we obtain

$$
\frac{\partial d}{\partial \boldsymbol{x}}(\boldsymbol{x})=\boldsymbol{B}_{d}(\boldsymbol{x}) \boldsymbol{a}_{d}
$$

where the array $\boldsymbol{B}_{d}$ contains the gradients of the finite element basis functions. When we interpolate the test functions in the same space as the trial functions the following system of equations is obtained from equation (24)

$$
\left[\int_{\Omega}\left[(1+\mathcal{F}) \boldsymbol{N}_{d}^{T} \boldsymbol{N}_{d}+4 \epsilon^{2} \boldsymbol{B}_{d}^{T} \boldsymbol{B}_{d}\right] \mathrm{d} V\right] \boldsymbol{a}_{d}=\left(\int_{\Omega} \mathcal{F} \boldsymbol{N}_{d}^{T} \mathrm{~d} V\right)
$$

The mesh dependent parameter $h$ in the definition of the driving force $\mathcal{F}$, equation (20), is related directly to the finite element mesh size. When the system (49) is solved for the coefficients $\boldsymbol{a}_{d}$, the approximate Dirac function of equation (25) can be expressed as

$$
\delta_{\epsilon}=\boldsymbol{a}_{d}^{T}\left[\frac{1}{4 \epsilon} \boldsymbol{N}_{d}^{T} \boldsymbol{N}_{d}+\epsilon \boldsymbol{B}_{d}^{T} \boldsymbol{B}_{d}\right] \boldsymbol{a}_{d}
$$

\subsection{Discretisation of the displacement fields}

For the discretisation of the displacement field and displacement jump field we use:

$$
\begin{aligned}
& \boldsymbol{u}=\boldsymbol{N}_{u} \boldsymbol{a}_{u} \rightarrow \operatorname{sym}\left(\frac{\partial \boldsymbol{u}}{\partial \boldsymbol{x}}\right)=\boldsymbol{B}_{u} \boldsymbol{a}_{u} \\
& \boldsymbol{v}=\boldsymbol{N}_{v} \boldsymbol{a}_{v} \rightarrow \operatorname{sym}(\boldsymbol{v} \otimes \boldsymbol{n})=\boldsymbol{B}_{v} \boldsymbol{a}_{v} \quad, \quad \frac{\partial \boldsymbol{v}}{\partial x_{n}}=\boldsymbol{G}_{v} \boldsymbol{a}_{v}
\end{aligned}
$$

It is noted that the tensorial quantities considered here are written in Voigt notation. Using this discretisation the following non-linear system of equations results

$$
\begin{aligned}
& \boldsymbol{f}_{\mathrm{int}, u}\left(\boldsymbol{a}_{u}, \boldsymbol{a}_{v}\right)=\boldsymbol{f}_{\mathrm{ext}, u} \\
& \boldsymbol{f}_{\mathrm{int}, v}\left(\boldsymbol{a}_{u}, \boldsymbol{a}_{v}\right)=\mathbf{0}
\end{aligned}
$$




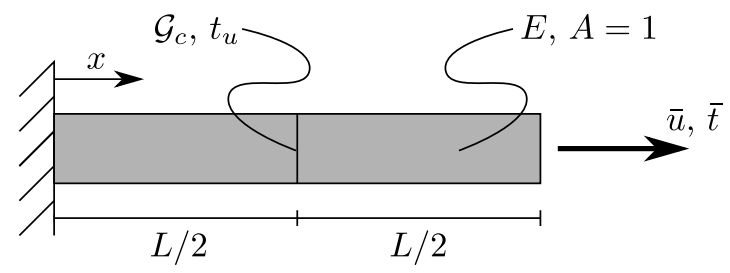

Figure 6. Schematic representation of a uniaxial rod with an elastic interface, $\mathcal{G}=\frac{1}{2} k \llbracket u \rrbracket^{2}$, or cohesive interface with parameters $\mathcal{G}_{c}$ and $t_{u}$

with the internal force vectors:

$$
\begin{aligned}
\boldsymbol{f}_{\mathrm{int}, u} & =\int_{\Omega} \boldsymbol{B}_{u}^{T} \boldsymbol{\sigma} \mathrm{d} V \\
\boldsymbol{f}_{\mathrm{int}, v} & =\int_{\Omega}\left(-\delta_{\epsilon} \boldsymbol{B}_{v}^{T} \boldsymbol{\sigma}+\delta_{\epsilon} \boldsymbol{N}_{v}^{T} \boldsymbol{t}(\boldsymbol{v})+\alpha \boldsymbol{G}_{v}^{T} \boldsymbol{G}_{v} \boldsymbol{a}_{v}\right) \mathrm{d} V
\end{aligned}
$$

Note that the non-linearity of equation (52) is a consequence of the non-linearity of the cohesive law, $t(v)$, equation (5). The system of equations (52) is solved using a standard incremental NewtonRaphson procedure with the tangent stiffness matrix

$$
\boldsymbol{K}=\left[\begin{array}{ll}
\boldsymbol{K}_{u u} & \boldsymbol{K}_{u v} \\
\boldsymbol{K}_{v u} & \boldsymbol{K}_{v v}
\end{array}\right]
$$

with

$$
\begin{aligned}
\boldsymbol{K}_{u u} & =\int_{\Omega} \boldsymbol{B}_{u}^{T} \boldsymbol{D} \boldsymbol{B}_{u} \mathrm{~d} V \\
\boldsymbol{K}_{u v}=\boldsymbol{K}_{v u}^{T} & =\int_{\Omega}-\delta_{\epsilon} \boldsymbol{B}_{u}^{T} \boldsymbol{D} \boldsymbol{B}_{v} \mathrm{~d} V \\
\boldsymbol{K}_{v v} & =\int_{\Omega}\left(\delta_{\epsilon}^{2} \boldsymbol{B}_{v}^{T} \boldsymbol{D} \boldsymbol{B}_{v}+\delta_{\epsilon} \boldsymbol{N}_{v}^{T} \frac{\partial \boldsymbol{t}}{\partial \boldsymbol{v}} \boldsymbol{N}_{v}+\alpha \boldsymbol{G}_{v}^{T} \boldsymbol{G}_{v}\right) \mathrm{d} V
\end{aligned}
$$

and $\boldsymbol{D}$ the Hookean tensor in Voigt notation.

\section{NUMERICAL SIMULATIONS}

In this section we study the performance of the cohesive phase-field formulation for a set of one and two-dimensional benchmark problems. First, we will consider the stability and approximation properties of the finite element discretisation for a one-dimensional test problem. Then, a delamination peel test is taken to demonstrate the applicability of the method in two dimensions. Finally, a first test case with a propagating interface is considered.

\subsection{Discretisation stability}

Since we consider a mixed finite element discretisation, equation (51), the stability of the discretisation can depend on the polynomial orders of the basis functions used for the discretisation of the various fields [29]. To study the discretisation stability we consider a one-dimensional domain of unit length with a modulus of elasticity $E=1$ and a centred elastic interface, see Figure 6 . The energy in the interface, with stiffness $k=1$, is taken as:

$$
\mathcal{G}=\frac{1}{2} k \llbracket u \rrbracket^{2}=\frac{1}{2} k v^{2}
$$

so that $t=k v$. The length scale parameter $\epsilon$ is taken equal to $L / 20$. 



Figure 7. Displacement field and stress field obtained using a discretisation based on piecewise linear polynomials for all fields

Figure 7 shows the solution for the displacement field and corresponding stress field for the case that all fields are discretised using piecewise linear basis functions. The solution of the stress field clearly reveals an oscillatory behaviour. This behaviour is explained by consideration of the elastic strain, which, in this one-dimensional case, is given by

$$
\varepsilon^{e}=\frac{\mathrm{d} u}{\mathrm{~d} x}-\delta_{\epsilon} v=\frac{\mathrm{d} u}{\mathrm{~d} x}-\left[\frac{d^{2}}{4 \epsilon}+\epsilon\left(\frac{\mathrm{d} d}{\mathrm{~d} x}\right)^{2}\right] v .
$$

Since $u$ is approximated by piecewise linear functions the strain distribution that derives from it is piecewise constant, see Figure 8 (left). When we consider the contribution to the elastic strain of the jump field, we observe that $\delta_{\epsilon}$ is approximated by piecewise quadratic polynomials and $v$ by piecewise linear functions. The jump is forced to be constant in the direction perpendicular to the discontinuity, which effectively makes the contribution of the jump field to the strain piecewise quadratic. The stress oscillations observed in Figure 7 (right) are a direct consequence of the mismatch in the approximation orders of the two strain contributions, see Figure 8 (left).

From equation (60) it is observed that when the polynomial order of the displacement field is taken equal to three, the orders of the strain contributions do match, see Figure 8 (right). In Figure 9 we observe that using the discretisation with piecewise linear basis functions for the phase field and displacement jump field and piecewise cubic polynomials for the displacement field indeed makes the stress oscillations vanish. The development of stable finite element schemes (including rigorous derivations) for the proposed formulation is a subject of further study.

\subsection{Cohesive fracture of a rod}

We again consider the one-dimensional rod of Figure 6, but now with a cohesive interface. The length of the rod is $L=1$ and the modulus of elasticity $E=1$. The interface energy is taken as [30]

$$
\mathcal{G}=\mathcal{G}_{c}\left[1-\left(1+\frac{\llbracket u \rrbracket}{v_{n}}\right) \exp \left(-\frac{\llbracket u \rrbracket}{v_{n}}\right)\right]
$$

with $v_{n}=\mathcal{G}_{c} /\left(t_{u} e\right), e=\exp (1)$, from which the traction follows as

$$
t=\mathcal{G}_{c} \frac{\llbracket u \rrbracket}{v_{n}^{2}} \exp \left(-\frac{\llbracket u \rrbracket}{v_{n}}\right) .
$$

The fracture energy and fracture strength are taken as $\mathcal{G}_{c}=1$ and $t_{u}=0.75$, respectively. The length scale parameter $\epsilon$ is taken as $\frac{L}{20}$ and the stabilization parameter $\alpha=0.1$.

We discretise the problem using third-order interpolation functions for the displacement field and first-order interpolation functions for the auxiliary field and for the phase field. The equilibrium 

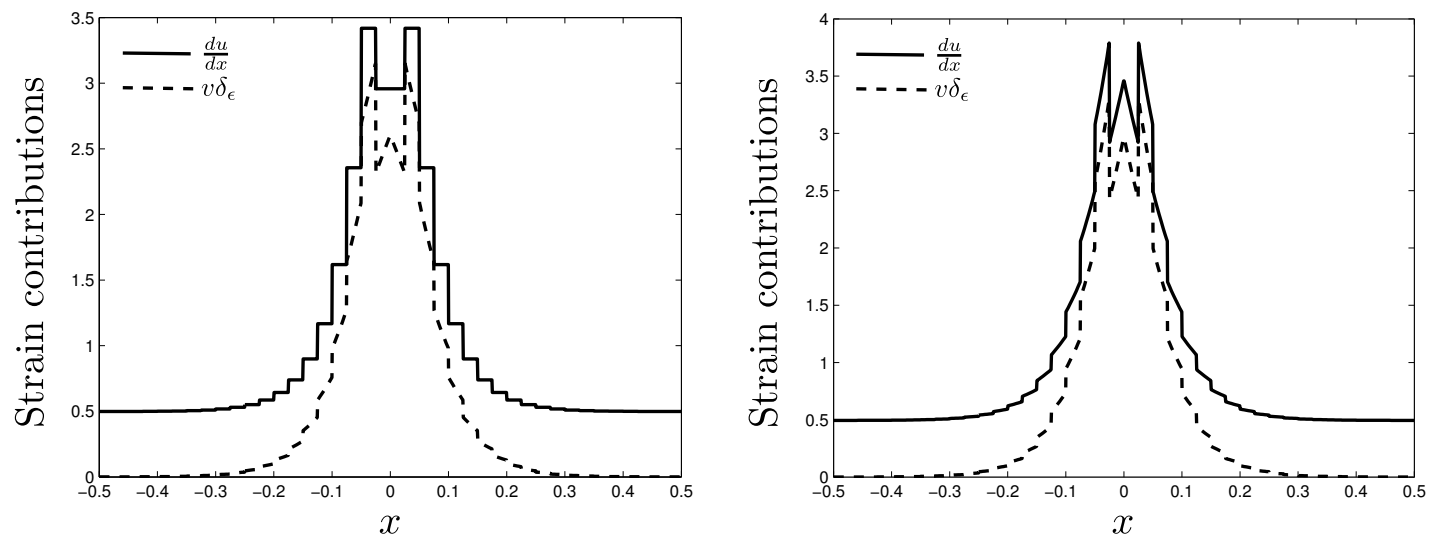

Figure 8. Strain contributions for a discretisation based on piecewise linear polynomials for all fields (left) and a discretisation in which the polynomial order of the displacement field is equal to three (right)
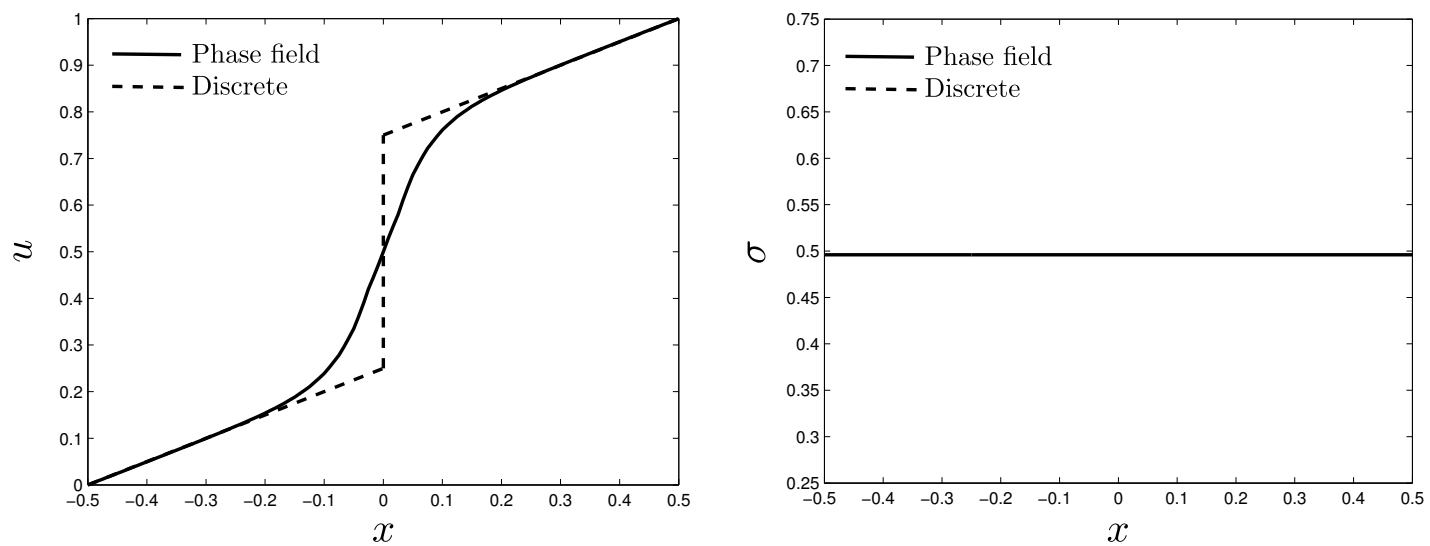

Figure 9. Displacement field and stress field obtained using a discretisation based on piecewise linear polynomials for the phase field and displacement jump field, and piecewise cubic polynomials for the displacement field

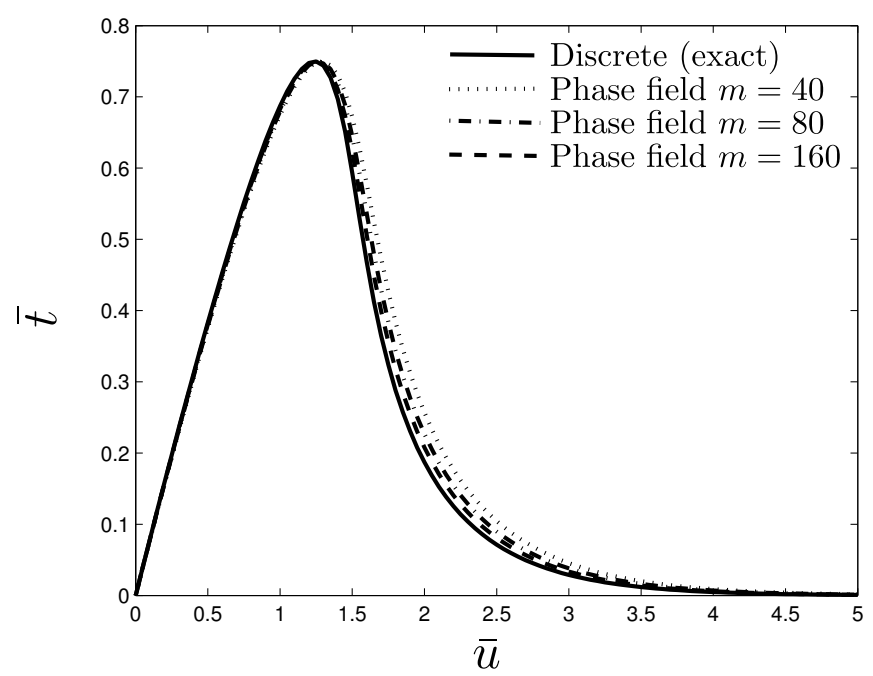

Figure 10. Force-displacement curve for the one-dimensional cohesive zone problem

path is traced using a displacement-controlled incremental iterative Newton-Raphson solver. The 


\begin{tabular}{|l||c|c|c|c|c|c|c|}
\hline$\alpha$ & $t_{u} \cdot 10^{-5}$ & $t_{u} \cdot 10^{-4}$ & $t_{u} \cdot 10^{-3}$ & $t_{u} \cdot 10^{-2}$ & $t_{u} \cdot 10^{-1}$ & $t_{u}$ & $t_{u} \cdot 10$ \\
\hline Stability & $\times$ & $\times$ & $\checkmark$ & $\checkmark$ & $\checkmark$ & $\checkmark$ & $\checkmark$ \\
\hline
\end{tabular}

Table I. Influence of the numerical parameter $\alpha$ on the stability of the results. Unphysical oscillations in the force-displacement curve are observed if $\alpha$ is chosen too small $(\times)$, whereas this curve is stable $(\checkmark)$ for sufficiently large $\alpha$

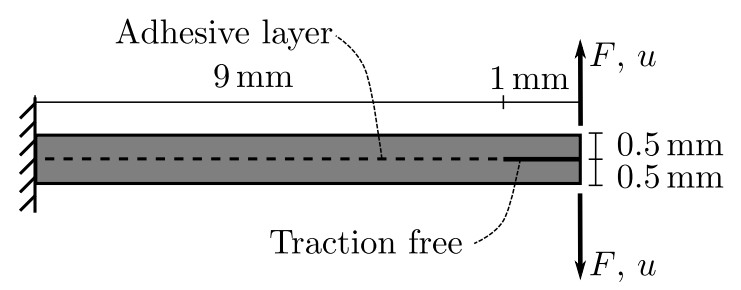

Figure 11. Schematic representation of the problem setup for the delamination peel test

displacement steps are taken equal to 0.05 . Figure 10 shows the computed response for various mesh refinments of the phase-field and compare it with the exact solution to the discrete problem. We observe that upon mesh refinement the phase-field model converges to the discrete solution.

As indicated in previous sections, the numerical parameter $\alpha$ is used to enforce the auxiliary displacement field to be constant perpendicular to the crack trajectory. In the case of the onedimensional problem considered here, this displacement jump field must be constant over the entire domain. As observed from equation (46) the parameter $\alpha$ can affect the local force balance and should therefore be selected appropriately. Based on equation (46) it appears to be natural to relate $\alpha$ to the fracture strength of a cohesive model. In Table I we list the results of a numerical investigation of the influence of $\alpha$. If $\alpha$ is chosen too small, i.e. less than $10^{-3}$ times the fracture strength, unphysical oscillations are observed in the force-displacement diagram, which can be traced back to local fluctuations in the displacement jump field. Taking $\alpha=0$ results in ill-conditioned tangent matrices which makes the results not trustworthy. If $\alpha$ is taken equal to $10^{-3}$ times the fracture strength or larger, stable results are obtained. Increasing the parameter $\alpha$ up to 10 times the fracture strength does not result in a visual effect on the force-displacement curve. Selecting the parameter $\alpha$ to be of the same order of magnitude as the fracture strength appears to be a good first estimate. The appropriateness of this choice can then be verified by numerically studying its influence.

\subsection{Delamination peel test}

To demonstrate the applicability of the phase-field formulation to cohesive fracture in a twodimensional case, we consider the delamination peel test shown in Figure 11. The test consists of two cantilever beams that are $10 \mathrm{~mm}$ long and $0.5 \mathrm{~mm}$ high, and are connected over $90 \%$ of their length by means of an adhesive layer. Upon increasing the externally applied loads, $F$, this adhesive layer will debond progressively. The bulk material is modelled as a linear isotropic material with a modulus of elasticity $E=100 \mathrm{MPa}$ and Poisson's ratio $\nu=0.3$. Plane-strain conditions are assumed. The behaviour of the adhesive layer is modelled using the Xu-Needleman decohesion relation [30], which, for mode-I crack opening resembles equation (61). The fracture strength and fracture energy are taken as, $t_{u}=1 \mathrm{MPa}$ and $\mathcal{G}_{c}=0.1 \frac{\mathrm{N}}{\mathrm{mm}}$, respectively. For the length scale parameter we take $\epsilon=0.05$, which corresponds to $5 \%$ of the total height of the specimen. The parameter $\alpha$ is taken equal to $1 \mathrm{MPa}$.

Following the conclusions regarding discretisation stability in the one-dimensional case, we use a mixed interpolation scheme consisting of third-order interpolation functions for the displacement field and first-order functions for the auxiliary field and for the phase field. To verify the correctness of this choice, we compare the stress field in the vertical direction around the traction free pre-crack with results obtained using first-order interpolation functions for all fields, see Figure 12. The stress field that is shown has been obtained after one small displacement step, so that non-linear effects 


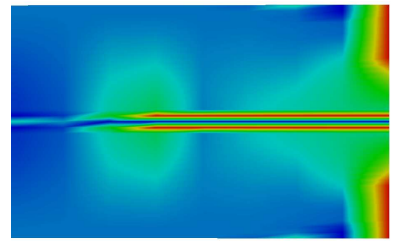

(a) Equal interpolation orders

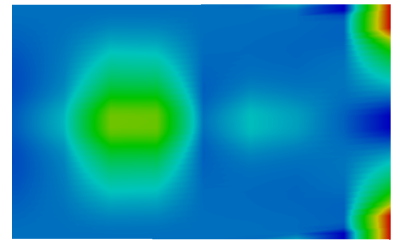

(b) Mixed interpolation orders

Figure 12. Stress field $\left(\sigma_{y y}\right)$ in the vicinity of the traction free interface after the first load step with (a) equal interpolation orders for all field variables, and (b) mixed interpolation orders

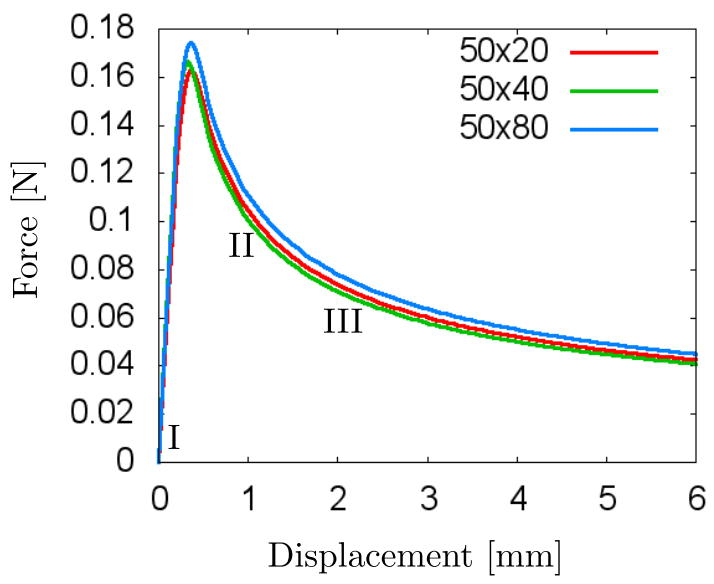

Figure 13. Force-displacement diagram for the delamination peel test computed for various meshes

related to the interface opening are negligible. As in the one-dimensional case, the proper selection of the interpolation orders is crucial.

Figure 13 gives the force-displacement curves obtained for different discretisations. All curves have been obtained using a displacement-controlled incremental-iterative solution procedure with displacement steps equal to $0.04 \mathrm{~mm}$. The results are objective with respect to the mesh size. Mesh refinement along the interface gives a similar picture. The results are also in good agreement with results reported in literature [31]. The roman numbers in Figure 13 refer to the three stages in the simulation shown in Figure 14. Evidently, the phase field does not change during the simulation, since there is an interface along the entire specimen right from the start. The stress field, given in the right part of Figure 14, shows how the delamination front progresses. Note that in this figure the interface is represented by a white line running through the specimen for the purpose of visualization.

\subsection{Peel test with propagating interface}

We now present a first result for a propagating crack. We again consider the peel test discussed in the preceding section, but now with only a traction-free pre-crack, see Figure 15. Upon increasing the load the crack is allowed to propagate into the bulk material. The material properties are taken the same as in the previous section. An exponentially decaying cohesive relation is used with the fracture energy identical to that in the previous section. The orders of the discretisation and the other numerical parameters are also taken identical to those in the previous section.

Since this contribution has focused on adhesive interfaces, the issue of crack nucleation and propagation was not addressed in Section 2. From the perspective of the development of a cohesive phase-field model it is necessary to develop a compatible nucleation/propagation model. This is not addressed here rigorously, but we demonstrate that with an ad-hoc model, meaningful results can be obtained. Our ad-hoc crack evolution model starts with the definition of a pre-crack, $\Gamma^{0}$, which 



I
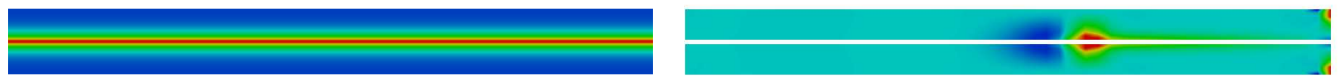

III
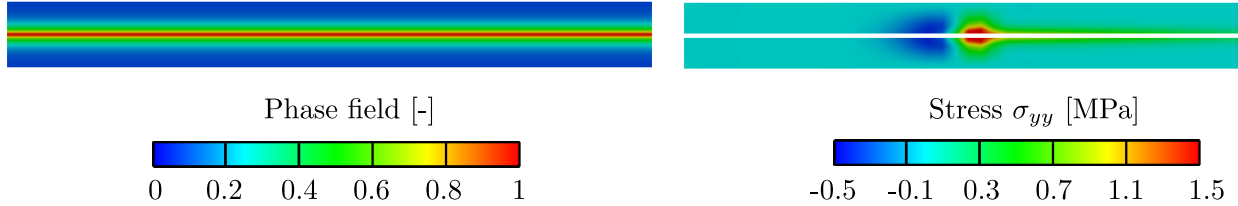

Figure 14. Phase field and $\sigma_{y y}$ stress field at three progressive stages in the delamination peel test

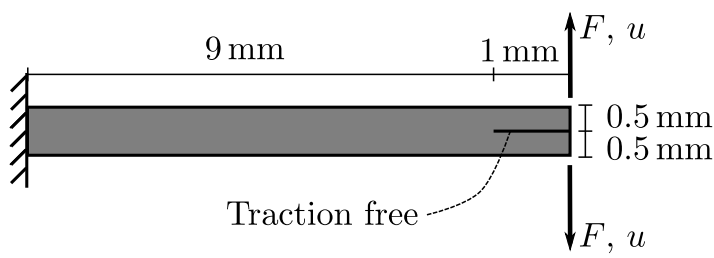

Figure 15. Schematic representation of the problem setup for the peel test with propagating discontinuity

is numerically represented by a set of positions and normal vectors sampled along the interface:

$$
\mathcal{S}^{0} \subset\left\{(\boldsymbol{x}, \boldsymbol{n}(\boldsymbol{x})) \in \Gamma^{0}\right\}
$$

If no pre-crack is available, the set $\mathcal{S}^{0}$ is empty. Using the point set $\mathcal{S}^{0}$, the driving force, equation (20) can however be evaluated at all integration points in the bulk. This allows for solving the phase-field problem, equation (49). Using the smeared crack represented by this field the displacements and displacement jumps can then be computed for the next time step through the solution of the non-linear set of equations (52). Upon convergence, say at time $t+\Delta t$, we evaluate the nucleation criterion in all integration points and extend the point set $\mathcal{S}^{t}$ of the previous time step with the points for which the nucleation criterion is now satisfied:

$$
\mathcal{S}^{t+\Delta t}=\mathcal{S}^{t} \cup\left\{\left(\boldsymbol{x}_{i}, \boldsymbol{n}_{i}\right) \mid \Sigma_{\text {nuc }}\left(\boldsymbol{\sigma}_{i}\right)>t_{u}, i \in \mathcal{I}_{\text {int }}\right\}
$$

In this expression $\Sigma_{\text {nuc }}\left(\sigma_{i}\right)>t_{u}$ is the nucleation criterion evaluated for the stresses $\sigma_{i}$ in all integration points of the set $\mathcal{I}_{\text {int }}$. The nucleation/propagation model should also yield a normal vector, $\boldsymbol{n}_{i}$, in all points where the criterion is met. An obvious choice to render this normal vector would be to use a maximum principal stress criterion as is commonly done in discrete descriptions of the cohesive zone model. In the present example we force the crack to remain straight by fixing the normal vectors in the required (i.c. upward) direction. Once a new set of points has been computed, the phase-field is recomputed by solving equation (49), and the displacements are updated by solving equation (52). The external loading is increased when no new nucleation/propagation points are found. For small load steps this usually happens after a single cycle of this staggered scheme.

In Figure 16 the force-displacement curves are shown for various discretisations. Clearly, the results are objective with respect to the discretisation. Also, the results are in excellent agreement with results reported in literature [31]. As in the previous section, the roman numbers refer to the three stages of the simulation shown in Figure 17. Note that the phase field now progresses during the simulation. As expected, the stress field evolves in a manner comparable to that of the adhesive interface discussed above. 


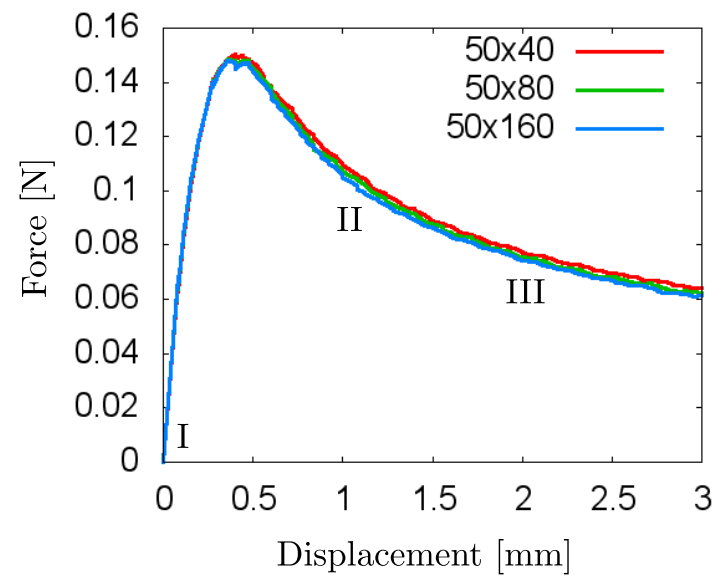

Figure 16. Force-displacement diagram for the peel test with propagating crack computed for various meshes

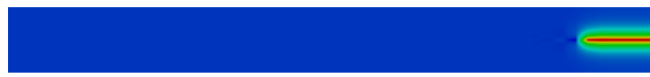

II

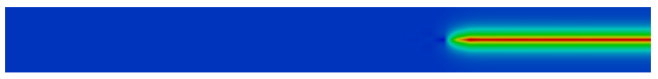

III
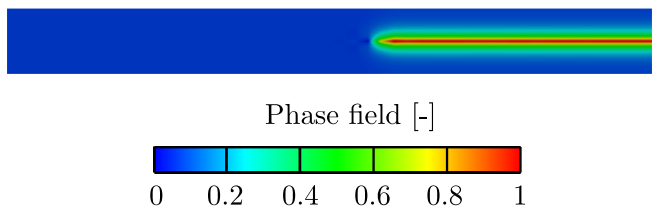
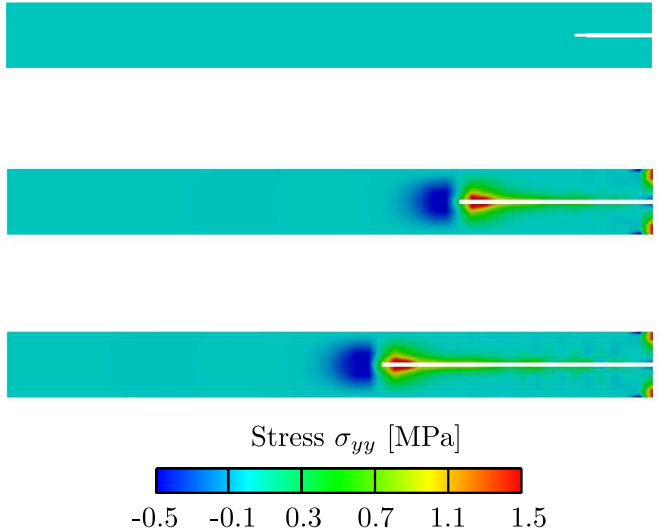

Figure 17. Phase field and $\sigma_{y y}$ stress field at three progressive stages in the peel test with propagating crack

\section{CONCLUDING REMARKS}

The derived phase-field model for cohesive fracture differs significantly from models available for brittle fracture. This is a direct consequence of the fact that the dissipative mechanism for cohesive fracture is different from that in brittle fracture. More specifically, in the cohesive case the crack opening must be available as a properly defined kinematic quantity, whereas in the brittle case the energy balance only requires a smeared description of internal discontinuity boundaries. For this reason, an auxiliary field is introduced to enable the incorporation of cohesive tractions during crack opening. Moreover, an elastic strain tensor is defined in the vicinity of the crack. Together with a standard linear isotropic constitutive relation this elastic strain tensor provides the contribution of the bulk material to the potential energy of the system. This elastic energy is balanced by a crack opening-dependent fracture energy term.

The model yields numerical results which are objective with respect to mesh refinement, and the results are in good agreement with results obtained using established discrete models. With respect to the simulation of adhesive fracture, an inconvenience of the model is the penalty term which enforces the auxiliary crack opening field to be constant in the direction orthogonal to the crack. Enforcing this constraint through a Lagrange multiplier field would eliminate the parameter $\alpha$, and can result in a simplification of the model.

The model discussed herein does not address the existence of weak discontinuities in the displacement field. The numerical simulations considered did not show problems with respect to the 
existence of jumps in the strain field, but these may, for example, occur when considering interfaces between materials with significantly different stiffnesses.

The model decouples the phase field evolution from the displacement field. In the case of adhesive interfaces this is a valid assumption, since the discontinuity boundaries are then constant throughout the simulation. As demonstrated, the decoupled model can be used in a staggered scheme to simulate crack propagation. The nucleation and propagation of cracks with an arbitrary topology should follow from the energy balance of a fully coupled model. The staggered scheme considered in this work is anticipated to be a special case of a fully coupled model.

\section{REFERENCES}

1. Ngo D, Scordelis AC. Finite element analysis of reinforced concrete beams. Journal of the American Concrete Institute 1967; 64: 152-163.

2. Rashid YR. Analysis of reinforced concrete pressure vessels. Nuclear Engineering and Design 1968; 7: $334-344$.

3. Ingraffea AR, Saouma V. Numerical modelling of discrete crack propagation in reinforced and plain concrete. In Fracture Mechanics of Concrete, pp. 171-225, Martinus Nijhoff Publishers: Dordrecht, 1985.

4. Camacho GT, Ortiz M. Computational modelling of impact damage in brittle materials. International Journal of Solids and Structures 1996; 33: 2899-2938.

5. Fleming M, Chu YA, Moran B, Belytschko T. Enriched element-free Galerkin methods for crack tip fields. International Journal for Numerical Methods in Engineering 1997; 40: 1483-1504.

6. Belytschko T, Black T. Elastic crack growth in finite elements with minimal remeshing. International Journal for Numerical Methods in Engineering 1999; 45: 601-620.

7. Moës N, Dolbow J, Belytschko T. A finite element method for crack growth without remeshing. International Journal for Numerical Methods in Engineering 1999; 46: 131-150.

8. Wells GN, Sluys LJ. A new method for modelling cohesive cracks using finite elements. International Journal for Numerical Methods in Engineering 2001; 50: 2667-2682.

9. Remmers JJC, de Borst R, Needleman A. A cohesive segments method for the simulation of crack growth. Computational Mechanics 2003; 31: 69-77.

10. de Borst R, Remmers JJC, Needleman A. Mesh-independent discrete numerical representations of cohesive-zone models. Engineering Fracture Mechanics 2006; 73: 160-177.

11. Moës N, Gravouil A, Belytschko T. Non-planar 3D crack growth by the extended finite element and level sets Part I: Mechanical model. International Journal for Numerical Methods in Engineering 2002; 53: 2549-2568.

12. Gravouil A, Moës N, Belytschko T. Non-planar 3D crack growth by the extended finite element and level sets Part II: Level set update. International Journal for Numerical Methods in Engineering 2002; 53: 2569-2586.

13. de Borst R, Gutiérrez MA. A unified framework for concrete damage and fracture models including size effects. International Journal of Fracture 1999; 95: 261-277.

14. de Borst R, Crisfield MA, Remmers JJC, Verhoosel CV. Non-linear Finite Element Analysis of Solids and Structures, Second Edition. Wiley: Chichester, 2012.

15. Pijaudier-Cabot G, Bažant ZP. Nonlocal damage theory. ASCE Journal of Engineering Mechanics 1987; 113: 1512 1533.

16. Peerlings RHJ, de Borst R, Brekelmans WAM and de Vree HPJ. Gradient-enhanced damage for quasi-brittle materials. International Journal for Numerical Methods in Engineering 1996; 39: 3391-3403.

17. Francfort GA, Marigo JJ. Revisiting brittle fracture as an energy minimization problem. Journal of the Mechanics and Physics of Solids 1998; 46: 1319-1342.

18. Bourdin B, Francfort GA, Marigo JJ. Numerical experiments in revisited brittle fracture. Journal of the Mechanics and Physics of Solids 2000; 48: 797-826.

19. Bourdin B, Francfort GA, Marigo JJ. The variational approach to fracture. Journal of Elasticity 2008; 91: 5-148.

20. Mumford D, Shah J. Optimal approximations by piecewise smooth functions and associated variational problems. Communications in Pure and Applied Mathematics 1989; 42: 577-685.

21. Miehe C, Hofacker M. Welschinger F. Thermodynamically consistent phase-field models of fracture: Variational principles and multi-field FE implementations. International Journal for Numerical Methods in Engineering 2010; 83: $1273-1311$.

22. Miehe C, Hofacker M, Welschinger F. A phase field model for rate-independent crack propagation: Robust algorithmic implementation based on operator splits. Computer Methods in Applied Mechanics and Engineering 2010; 199: 2765-2778.

23. Hofacker M, Miehe C. A phase field model of dynamic fracture: Robust field updates for the analysis of complex crack patterns. International Journal for Numerical Methods in Engineering 2012; 93: 276-301.

24. Bourdin B, Larsen CJ, Richardson C. A time-discrete model for dynamic fracture based on crack regularization. International Journal of Fracture 2011; 168: 133-143.

25. Borden MJ, Verhoosel CV, Scott MA, Hughes TJR, Landis CM. A phase-field description of dynamic brittle fracture. Computer Methods in Applied Mechanics and Engineering 2012; 217-220: 77-95.

26. de Borst R, Benallal A, Heeres OM. A gradient-enhanced damage approach to fracture. Journal de Physique IV 1999; C6: 491-502.

27. Comi C. Computational modelling of gradient-enhanced damage in quasi-brittle materials. Mechanics of Cohesivefrictional Materials 1999; 4: 17-36.

28. Geers MGD, de Borst R, Brekelmans WAM, Peerlings RHJ. Strain-based transient-gradient damage model for failure analyses. Computer Methods in Applied Mechanics and Engineering 1998; 160: 133-153. 
29. Auricchio F, Brezzi F, Lovadina C. Mixed Finite Element Methods. In: Encyclopedia of Computational Mechanics, Wiley, 2004.

30. Xu XP, Needleman A. Void nucleation by inclusion debonding in a crystal matrix. Modelling and Simulation in Materials Science and Engineering 1993; 1: 111132.

31. Remmers JJC. Discontinuities in materials and structures: a unifying computational approach. PhD thesis Delft University of Technology, 2006 